\section{JTI}

JOURNAL OF

TRAUMA AND INJURY
Received: December 16, 2019

Revised: April 20, 2020

Accepted: June 25, 2020

\section{Correspondence to}

Sun Kyun Ro, MD, PhD

Department of Thoracic and Cardiovascular Surgery, Hanyang University Guri Hospital, Hanyang University College of Medicine, 222 Wangsimni-ro, Seong-

dong-gu, Seoul 04763, Korea

Tel: +82-31-560-2305

Fax: +82-31-565-2264

E-mail: skro@hanyang.ac.kr
(1)

J Trauma Inj 2020;33(2):124-127

https://doi.org/10.20408/jti.2019.041

\title{
Spontaneously Resolved Lumbar Artery Injury after Blunt Trauma
}

\author{
Seung Hyuk Nam, $\mathrm{MD}^{1}$, Je Il Ryu, MD, $\mathrm{PhD}^{2}$, Jin Hwan Cheong, $\mathrm{MD}, \mathrm{PhD}^{2}$, \\ Ki-Chul Park, MD, $\mathrm{PhD}^{3}$, Sun Kyun Ro, MD, $\mathrm{PhD}^{1}$ \\ ${ }^{1}$ Department of Thoracic and Cardiovascular Surgery, Hanyang University Guri Hospital, \\ Hanyang University College of Medicine, Seoul, Korea \\ ${ }^{2}$ Department of Neurosurgery, Hanyang University Guri Hospital, Hanyang University \\ College of Medicine, Seoul, Korea \\ ${ }^{3}$ Department of Orthopedic Surgery, Hanyang University Guri Hospital, Hanyang \\ University College of Medicine, Seoul, Korea
}

Major bleeding caused by vascular injuries of the abdominal aorta or its branches after blunt trauma often leads to mortality or major morbidity. We report a case that lumbar artery injury following blunt trauma was spontaneously resolved without any surgical or interventional treatment. Lumbar artery injury after blunt trauma could be treated conservatively without surgical or interventional treatment in a selected case. When an aortic or its branch injury was suspicious, diagnostic angiograms in the setting of interventional treatment may be helpful to decide an appropriate treatment modality.

Keywords: Vascular injuries; Blunt injuries; Angiography

\section{INTRODUCTION}

Abdominal vascular injuries may be caused by blunt trauma with rapid deceleration in motor vehicle collisions [1]. Of such vascular injuries, lumbar artery injury (LAI) often leads to mortality and major morbidity due to severity of vascular trauma per se or combined severe trauma [2]. Most studies concerning LAI have been announced as case reports or series. Referring to those studies, LAIs were mostly treated with endovascular procedures such as catheter embolization since surgical intervention often fails to identify bleeding sources, complicating further bleeding [3]. Additionally, more accurate diagnosis of the LAI should be necessary to decide an appropriate treatment modality, because it sometimes mimics abdominal aortic injury or could be even neglected. We reported a case that LAI following blunt trauma was spontaneously re(http://creativecommons.org/licenses/by-nc/4.0/) which permits unrestricted noncommercial use, distribution, and pISSN 1738-8767 
solved without any surgical or interventional treatment.

\section{CASE REPORT}

A 63-year old female visited our emergency room for blunt trauma caused by vehicle accident. She complained of pain in the abdomen, the right chest wall and the left forearm. Vital instability was not noted, and the initial blood pressure was $180 / 100 \mathrm{mmHg}$ on arrival at the emergency room. Focused assessment with sonography for trauma and imaging studies were performed to confirm further concealed trauma. On abdominal computed tomograms, large amount of hematoma adjacent to the infrarenal abdominal aorta with extravasation of contrast material was noted (Fig. 1). Lowering the blood pressure with intravenous beta blockade and preparing blood products for transfusion, she was urgently transferred to the catheterization room to rule out and treat for vascular injury of the abdominal aorta or its branches. On diagnostic angiograms, extravasation of contrast material was not noted, and the abdominal aortic wall seemed to be intact (Fig. 2). We decided to treat her conservatively with serial image follow-up. On the retrospective review of the initial computed tomograms, the LAI was suspected (Fig. 1B). On the 10th hospital day, decrement of the hematoma adjacent to the abdominal aorta was noted without any evidence of further active bleeding on the computed tomographic aortograms (Fig. 3A). She was discharged without any complication and followed up for three months (Fig. 3B).

\section{DISCUSSION}

LAI caused by blunt trauma is uncommon and is usually associated with severe abdominal injuries including intraabdominal organ, spinal or pelvic injuries [2]. Isolated LAI without other abdominal injuries is rare. According to prior reports, most of the abdominal vascular injuries by blunt trauma resulted from motor vehicle crashes [1]. Rapid deceleration in motor vehicle collisions may cause two different types of abdominal vascular injuries; avulsion of small branches from major vessels with subsequent hemorrhage and intimal tear with secondary thrombosis of the lumen [1]. This current case seems to be associated with the former mechanism leading to subsequent hemorrhage. Additionally, direct blunt force to the abdomen or back may be the predominant mechanism of abdominal vascular injury, in association with seat-belt use $[4,5]$.

Clinical manifestations of LAI show various spectrum according to its extent. Sometimes it could be resolved spontaneously with conservative treatment just like our
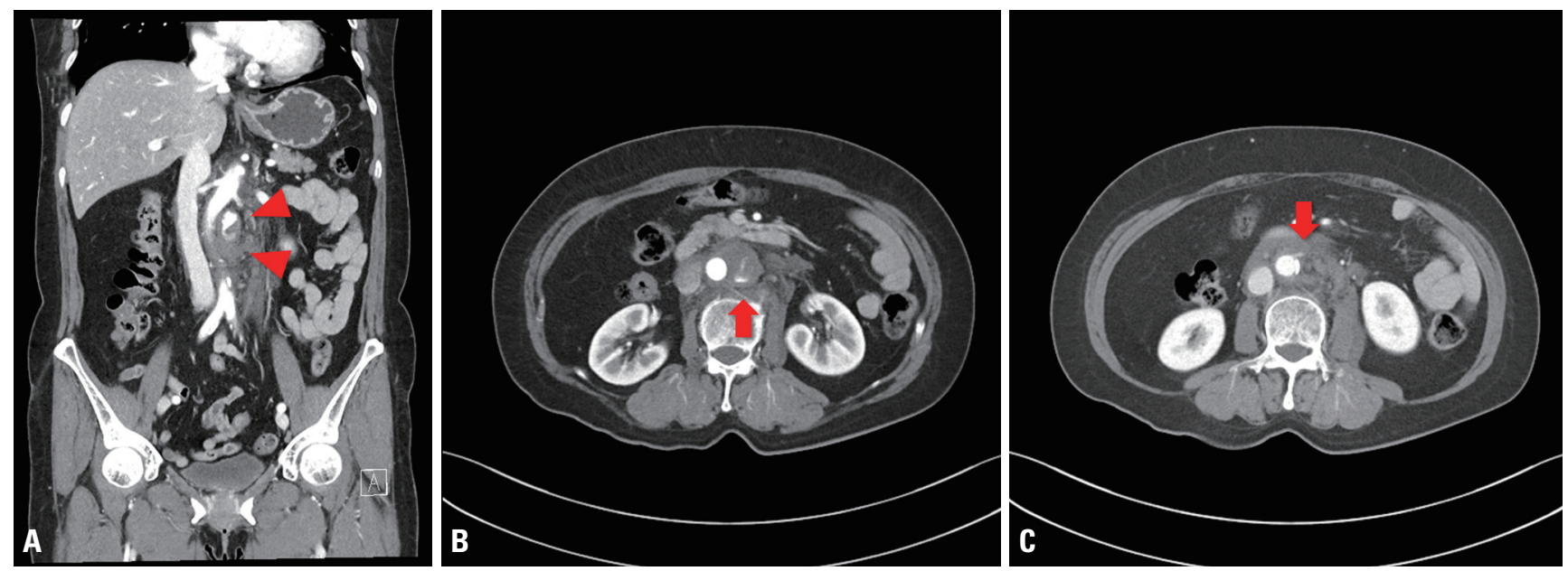

Fig. 1. Extravasation of contrast material adjacent to the infrarenal abdominal aorta on abdominal computed tomograms. (A) Arrow heads indicate extravasation of contrast with hematoma on the left side of the infrarenal abdominal aorta. (B) Contrast leakage from lumbar artery was suspected on an axial view (arrow). (C) The inferior mesenteric artery was intact without evidence of contrast leakage on an axial view (arrow). 


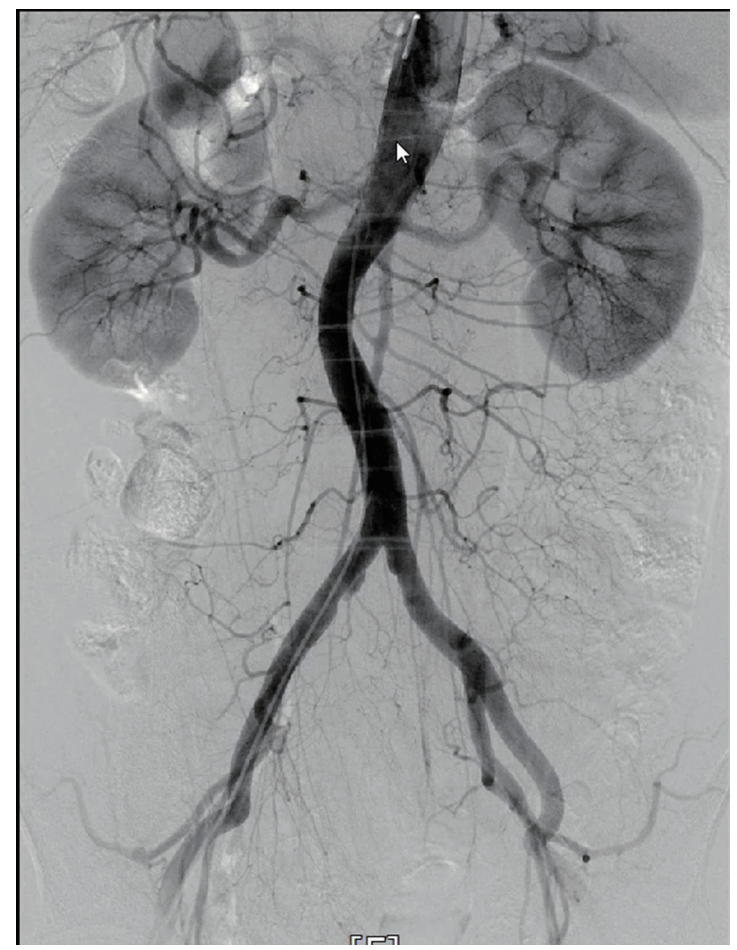

Fig. 2. Diagnostic angiograms showed no extravasation of contrast material and intact abdominal aortic wall.

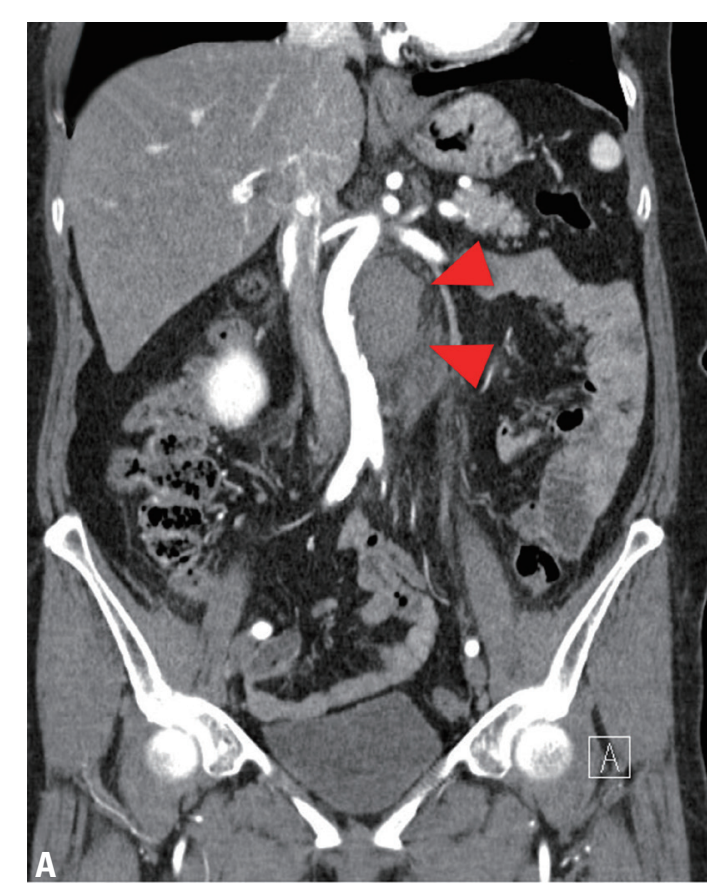

case, and sometimes it could lead to more critical conditions necessitating further treatment [6]. It is difficult to decide treatment modality based solely on the initial imaging study. Not only vital status but also consecutive imaging study may be helpful to decide an appropriate treatment option. In this current case, initial computed tomograms showed suspicious ongoing vascular injury of the abdominal aorta or its branches; however, active bleeding was not shown any more on consecutive angiograms. Especially, patient's vital stability was maintained while staying in the emergency room and that would be noteworthy. However, even stable or asymptomatic patients could be later diagnosed as lumbar artery pseudoaneurysm [6]. Therefore, follow-up imaging studies should be necessary not to miss detection of delayed pseudoaneurysm. In our case, the patient has been followed up for 3 months after hospital discharge and any evidence of lumbar artery pseudoaneurysm was not noted on the latest exams. Optimal duration of imaging follow-ups was not well established; however, imaging studies on the second or third day, 1, 6, and 12 months after the initial study were reasonable referring to the other guidelines

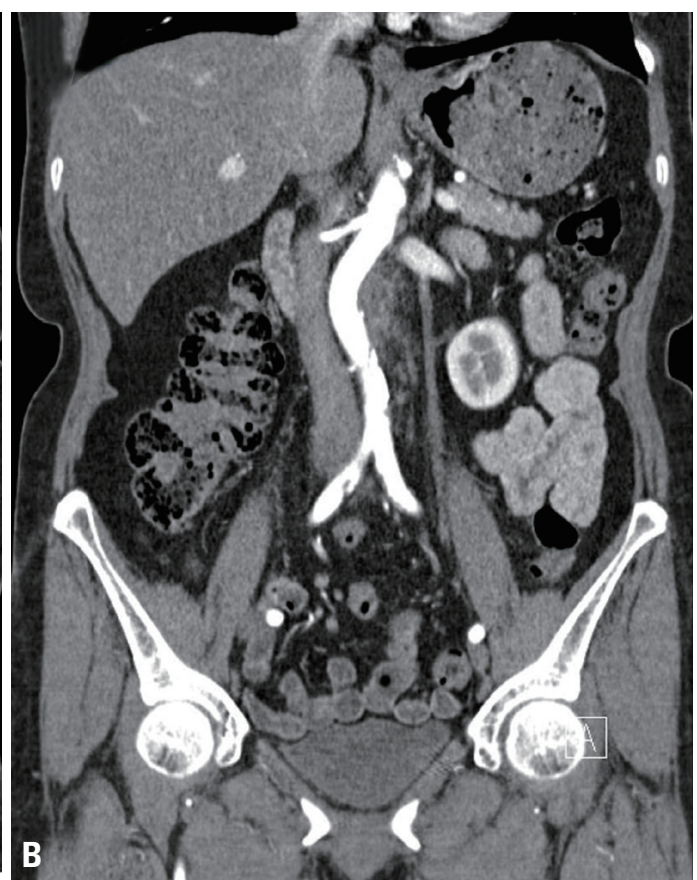

Fig. 3. Follow-up computed tomograms. (A) The size of hematoma adjacent to the abdominal aorta decreased without any evidence of further ongoing bleeding on the 10th hospital day (arrow heads). (B) Hematoma adjacent to the abdominal aorta was nearly disappeared three months after blunt trauma. 
concerning aortic diseases [7].

Clinical outcomes of such vascular injury are often devastating. Surgical exploration for LAI is challenging due to difficulty in proximal control and combined injuries [3]. Recently, endovascular interventions were preferred to surgical interventions to improve clinical outcome. Despite such effort, the mortality of abdominal vascular injuries including lumbar artery injury has been reported to be still high and optimal treatment modality, like surgical/ endovascular approach, is determined by several factors, like injury pattern, hemodynamic instability, institutional resources, etc $[2,4]$. Nevertheless, diagnostic angiography and subsequent endovascular treatment for LAI seem to be a good option, especially after stabilization of vital status [8].

Stable LAI by blunt trauma could be treated conservatively without surgical or interventional treatment in a selected case. When an aortic or its branch injury was suspicious, diagnostic angiograms in the setting of interventional treatment may be helpful to decide an appropriate treatment option.

\section{REFERENCES}

1. Moore EE, Feliciano DV, Mattox KL. Abdominal vascular injury. In: Asensio JA, Feliciano DV, eds. Trauma. 8th ed. New York: McGraw-Hill;2016:651-76.

2. Yuan KC, Hsu YP, Wong YC, Fang JF, Lin BC, Chen HW. Management of complicated lumbar artery injury after blunt trauma. Ann Emerg Med 2011;58:531-5.
3. Domenicucci M, Ramieri A, Lenzi J, Fontana E, Martini S. Pseudo-aneurysm of a lumbar artery after flexion-distraction injury of the thoraco-lumbar spine and surgical realignment: rupture treated by endovascular embolization. Spine (Phila Pa 1976) 2008;33:E81-4.

4. de Mestral C, Dueck AD, Gomez D, Haas B, Nathens AB. Associated injuries, management, and outcomes of blunt abdominal aortic injury. J Vasc Surg 2012;56:656-60.

5. Roth SM, Wheeler JR, Gregory RT, Gayle RG, Parent FN 3rd, Demasi R, et al. Blunt injury of the abdominal aorta: a review. J Trauma 1997;42:748-55.

6. Vashisht S, Bachhal V, Kumar V, Kumar D. Lumbar artery pseudoaneurysm following blunt trauma: A case report and literature review. Trauma Case Rep 2019;23:100232.

7. Hiratzka LF, Bakris GL, Beckman JA, Bersin RM, Carr VF, Casey DE Jr, et al. 2010 ACCF/AHA/AATS/ACR/ASA/SCA/SCAI/ SIR/STS/SVM guidelines for the diagnosis and management of patients with thoracic aortic disease. A report of the American College of Cardiology Foundation/American Heart Association task force on practice guidelines, American Association for Thoracic Surgery, American College of Radiology, American Stroke Association, Society of Cardiovascular Anesthesiologists, Society for Cardiovascular Angiography and Interventions, Society of Interventional Radiology, Society of Thoracic Surgeons, and Society for Vascular Medicine. J Am Coll Cardiol 2010;55:e27129.

8. Hagiwara A, Murata A, Matsuda T, Matsuda H, Shimazaki S. The usefulness of transcatheter arterial embolization for patients with blunt polytrauma showing transient response to fluid resuscitation. J Trauma 2004;57:271-6; discussion 6-7. 